DOI: 10.25100/eg.v0i22.11320

Espacios y Territorios

\title{
Influencia antrópica en el patrón espacial de la vegetación Leopardo (Leopard Bush) en La Guajira Colombiana ${ }^{1}$
}

\section{Anthropic influence in the spatial pattern of the Leopard Bush in the Colombian Guajira}

\author{
José Eduardo Fuentes Delgado \\ Departamento de Geografía. Universidad del Valle. Cali, Colombia. \\ jose.fuentes@correounivalle.edu.co | 0000-0003-1233-4924
}

\begin{abstract}
Para citar este artículo: Fuentes Delgado, J. (2021). Influencia antrópica en el patrón espacial de la vegetación Leopardo (Leopard Bush) en La Guajira Colombiana. Entorno Geográfico, (22), 21-35.
\end{abstract}

https://doi.org/doi:10.25100/eg.v0i22.11320

\section{Resumen}

Una revisión reciente de la estructura de los ecosistemas de las zonas áridas apoya la idea de que la vegetación está conformada comúnmente por dos patrones espaciales denominados "arbusto Tigre" y "arbusto Leopardo". Se plantea que la distribución y la composición del patrón Leopardo es originada por la interacción entre aspectos antrópicos y biofísicos. La presente investigación tiene como objetivo investigar el patrón Leopardo en la península de La Guajira, se implementó un enfoque biofísico que integra tecnologías de SIG y Percepción Remota. Se utilizó información recabada en campo, imágenes de satélite y modelos de elevación digitales para producir mapas de distribución vegetal. El análisis de los nutrientes del suelo mostró cómo varían los valores de $\mathrm{N}, \mathrm{P}$, $\mathrm{K}$ en los suelos desnudos y en las islas de fertilidad conformadas por la vegetación donde los cactus forman parte del patrón, siendo reportados por primera vez. El patrón Leopardo es el resultado de unas características específicas que limitan las formas de vida vegetales, al limitarse el desarrollo a solo ciertas especies resistentes, éstas conforman comunidades y a su vez generan estrategias para optimizar los recursos disponibles. En el patrón Leopardo de la Guajira los suelos compactados y los espacios fragmentados por la influencia antrópica mostraron ser uno de los principales factores en la heterogeneidad resultante.

Palabras clave: Aridez, SIG, Isla de fertilidad, Satélite, Vegetación.

\begin{abstract}
A recent review of the structure of arid zone ecosystems supports the idea that vegetation is commonly made up of two spatial patterns called "Tiger bush" and "Leopard bush". It is suggested that the distribution and composition of the Leopard pattern is caused by the interaction between anthropic and biophysical aspects. The present research aims to investigate the Leopard pattern in the La Guajira peninsula, a biophysical approach was implemented that integrates GIS and Remote
\end{abstract}

\footnotetext{
${ }^{1}$ Este estudio estuvo enmarcado dentro de un proyecto de doctorado en la convocatoria 568 de 2012 doctorados internacionales financiado por COLCIENCIAS.

(c) (i) (-)

Esta obra está bajo licencia internacional Creative Commons Reconocimiento-NoComercial-CompartirIgual 4.0.
} 
Sensing technologies. Information collected in the field, satellite images, and digital elevation models were used to produce plant distribution maps. The analysis of the soil nutrients showed how the values of $\mathrm{N}, \mathrm{P}, \mathrm{K}$ vary in bare soils and in the fertility islands formed by the vegetation where the cacti are part of the pattern, being reported for the first time. The Leopard pattern is the result of specific characteristics that limit plant life forms, by limiting development to only certain resistant species, these make up communities and in turn generate strategies to optimize available resources. In the Leopard pattern of La Guajira, compacted soils and spaces fragmented by anthropic influence were shown to be one of the main factors in the resulting heterogeneity.

Keywords: Aridity, GIS, Island of fertility, Satellite, Vegetation.

Recibido: 22 de octubre de 2020

Aceptado: 29 de abril de 2021

\section{Introducción}

Las tierras secas cubren aproximadamente el $41 \%$ de la superficie terrestre del planeta y más de 2000 millones de personas, alrededor del $35 \%$ de la población mundial, habitan en ellas. Los ecosistemas y las tierras secas en Colombia se calculan alrededor del 16,9 \% del total del país según el Instituto de Hidrología, Meteorología y Estudios Ambientales de Colombia (IDEAM, 2005). En particular, el departamento de la Guajira en su parte norte es un área poblada casi en su totalidad por comunidades aborígenes de la etnia Wayúu, las cuales han tenido la capacidad de adecuarse a los ambientes áridos en donde se ubicaron desde hace 5000 años atrás. Las condiciones climáticas en el entorno geográfico de la península de la Guajira se distinguen de las del resto de Colombia por no tener estaciones térmicas, predominar los fuertes vientos, por tener una precipitación irregular a lo largo del año y temperaturas medias anuales que pueden llegar a $33^{\circ} \mathrm{C}$ (IDEAM, 2005). El entendimiento de los patrones de vegetación en zonas áridas es importante en la conservación de suelos y en la lucha contra el cambio climático. Durante la década de los años cincuenta, Meigs (1953) "estudio la distribución de las zonas áridas y semiáridas a nivel mundial, encontró evidencias de patrones espaciales a nivel subregional y local, donde la vegetación de zonas secas conforma patrones regulares" (pp. 3), siendo observados en los primeros vuelos de fotografía aérea de zonas como Somalia, Sudan y Australia central (Macfadyen, 1950; Slatyer, 1959; Worral, 1959; Boaler \& Hodge, 1962; Wickens \& Collier, 1971).

Con el constante avance en la disponibilidad de información cartográfica y satelital de alta resolución espacial, se han podido analizar las zonas áridas con un mayor detalle. Los patrones espaciales regulares de la vegetación consisten en una cobertura vegetal de porte bajo que aparentemente ha sido fragmentada, posteriormente a la perturbación en dichas áreas la cobertura subsecuente revela áreas sin cobertura o con un suelo desnudo, y se muestra zonas de vegetación espaciadas de manera regular, la vegetación baja se intercala con vegetación de un porte más alto, estas áreas generalmente son homogéneas en la composición del material parental, del suelo y del clima.

Distintos autores soportan la teoría de que, en los ecosistemas áridos del mundo, la vegetación es distribuida de manera heterogénea y se subdivide en dos fases compuestas de parches con vegetación espaciada de manera regular; uno con cobertura alta y otro con cobertura baja o sin ninguna cobertura (Aguiar \& Sala, 1999; Rietkerk \& van de Koppel ,2008; Cheng et al., 2010). El llamado patrón Tigre (Tiger Bush) es caracterizado por presentar parches de vegetación, que conforman rayas o bandas de 
vegetación intercalada con espacios con muy poca vegetación o sin ella, pueden tener dimensiones que abarcan franjas con un largo desde los 30 a los $400 \mathrm{~m}$ y con un ancho desde 10 a los $50 \mathrm{~m}$ (Figura 1a). El patrón denominado Leopardo (Leopard Bush) se caracteriza porque presenta formas de parches o manchas de vegetación intercaladas con áreas espaciadas con poca cobertura o suelo desnudo, las dimensiones de este abarcan largos desde $1 \mathrm{~m}$ a los $10 \mathrm{~m}$ y dimensiones a lo ancho en formas variables desde los 2 a $50 \mathrm{~m}$ (Figura 1b).

Fuente: Elaboración propia con datos de Space imaging - Google Earth

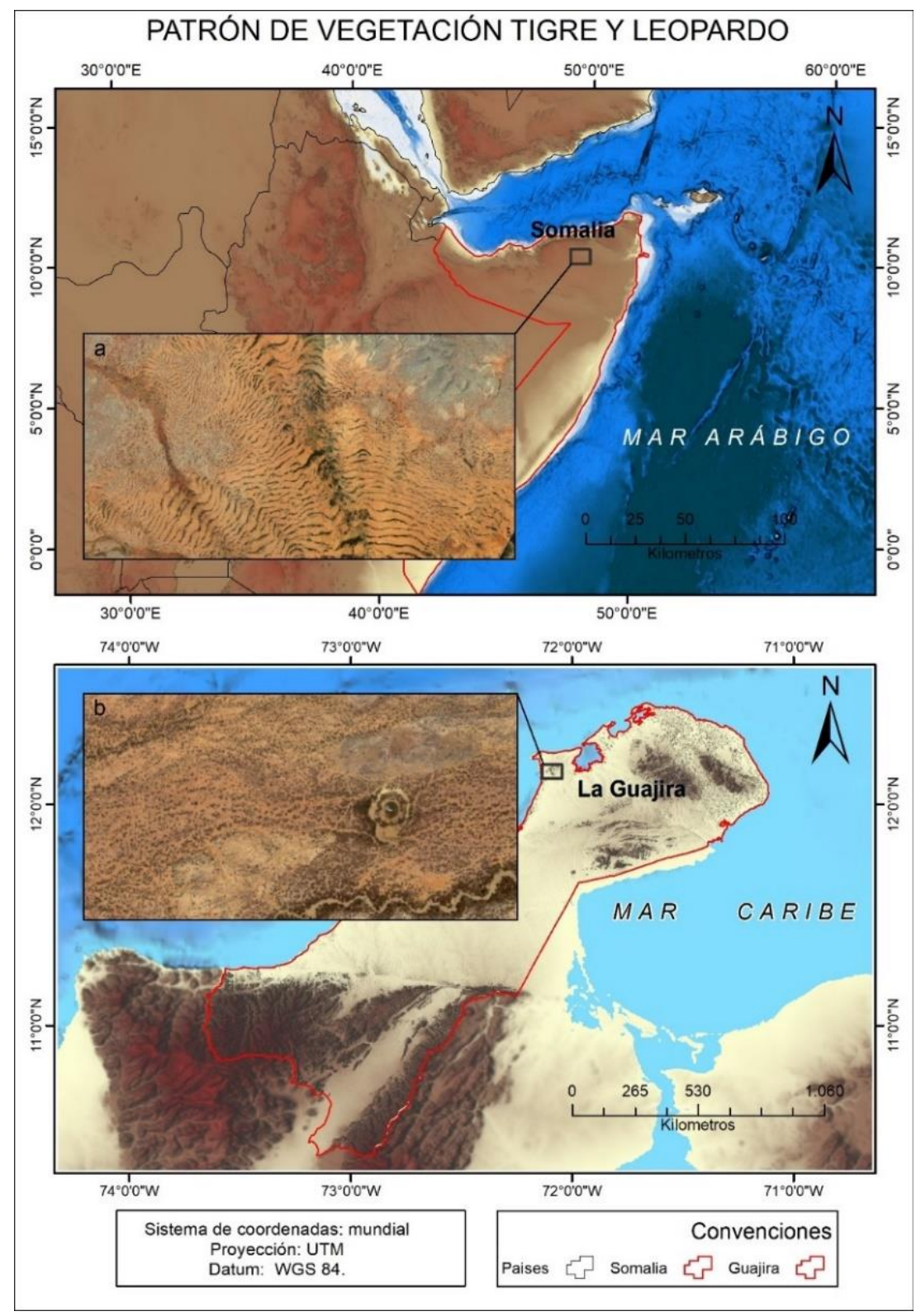

Figura 1: Patrón de vegetación Tigre (a) Somalia y Patrón de vegetación Leopardo (b) La Guajira, Colombia

Dentro de la vegetación se infiere que existe un mayor potencial para el desarrollo de las plantas por la presencia del efecto de isla de fertilidad (Schlesinger et al. 1990; Garcia-Moya \& McKell 1970), 
y la teoría de islas (MacArthur, 1967) en cuanto a la dinámica y equilibrio de los ecosistemas que conforman los patrones. Los estudios que analizan el patrón Leopard Bush son menores en cantidad si se compara contra el patrón Tiger Bush, gran parte de los estudios se fundamentan en análisis numéricos con modelos computacionales (Borgogno et al., 2009). El llegar a una conclusión clara del porqué de los patrones " Tigre Bush "y "Leopardo Bush", cómo se conforman y desarrollan ha sido difícil, muchas de las hipótesis en la bibliografía apuntan a que el desarrollo y la formación de estos patrones están relacionados con el efecto del fuego, el pastoreo de ungulados, las condiciones anisotrópicas (la pendiente), y otros factores como la diseminación de corta y larga distancia, además de la competitividad entre especies de la vegetación (Cheng et al., 2010). El estudio de patrones vegetales en zonas áridas, en especial el patrón Tigre, ha pasado por diversas etapas en los últimos cincuenta años. En las últimas dos décadas con el aumento en la capacidad de procesamiento y los avances en modelamiento numérico han permitido que el estudio de patrones inicie un período de "simulación" para tratar de entender su comportamiento y complejidad desde el punto de vista matemático (Tabla 1). Hasta la fecha, la mayoría de los modelos matemáticos utilizan información teórica referida al patrón Tigre y Leopardo, se centran en simulación de las rayas perpendiculares a la dirección del flujo en sistemas semiáridos.

En la actualidad no existe un consenso específico sobre la formación del patrón Tigre y Leopardo, principalmente por el número de hipótesis y puntos de vista sobre su formación y desarrollo. Por lo tanto, los patrones en mancha pueden ser mucho más ampliamente distribuidos de lo que parece desprenderse de la existente literatura, y como el patrón Leopardo, puede tener una amplia distribución mundial (Couteron \& Lejeune 2001; Fuentes et al., 2016). Las primeras referencias al patrón Leopardo en América se reportaron en la Patagonia Argentina (Sala \& Aguiar, 1996; Aguiar \& Sala, 1999) y posteriormente en Estados Unidos (Bestelmeyer et al., 2006; Yetemen et al., 2010; Bordeu et al., 2016). Sin embargo, es importante destacar que no se dispone de información previa sobre investigaciones que caractericen los patrones Leopardo en Colombia.

Tabla 1. Simulación de patrones en referencias bibliográficas

\begin{tabular}{|l|c|c|c|c|c|}
\hline Lugar & Continente & Patrón & Datos & Referencia & Modelo \\
\hline Nigeria & África & Bandas & Campo & (Blyth y Harding 1995) & Numérico \\
\hline Australia & Australia & Bandas & Simulación & (Dunkerley, 1997) & Celular autómata \\
\hline Mundial & Mundial & Bandas & Simulación & (Lefever \& Lejeune 1997) & Numérico \\
\hline Burkina Faso & África & Bandas y Manchas & $\begin{array}{c}\text { Simulación } \\
\text { Campo }\end{array}$ & (Lejeune et al., 1999) & Modelo Isotropico \\
\hline Burkina Faso & África & Bandas & $\begin{array}{c}\text { Simulación } \\
\text { Campo }\end{array}$ & (Rietkerk et al., 2000) & Numérico \\
\hline Burkina Faso & África & Bandas y Manchas & $\begin{array}{c}\text { Simulación } \\
\text { Campo }\end{array}$ & (Couteron \& Lejeune 2001) & Numérico \\
\hline Mundial & Mundial & Bandas & Simulación & (HilleRisLambers et al., 2001) & Numérico \\
\hline Mundial & Mundial & Bandas y Manchas & Simulación & (Gilad et al., 2004) & Numérico \\
\hline Mundial & Mundial & Bandas & Simulación & (Ursino, 2005) & Numérico \\
\hline Mundial & Mundial & Bandas & Simulación & (Saco et al., 2007) & Numérico \\
\hline Nigeria & África & Manchas & Simulación & (Barbier et al., 2008) & Numérico \\
\hline Mundial & Mundial & Bandas y Manchas & Simulación & (Manor y Shnerb 2008) & Numérico \\
\hline Mundial & Mundial & Bandas & Simulación & (Liu et al., 2008) & Numérico \\
\hline Mundial & Mundial & Bandas y Manchas & Simulación & (Kefi et al., 2008) & Numérico \\
\hline Chile & América & Bandas & Simulación & (Borthagaray et al., 2010) & Numérico \\
\hline
\end{tabular}




\begin{tabular}{|l|c|c|l|c|c|}
\hline Lugar & Continente & Patrón & Datos & Referencia & Modelo \\
\hline Mundial & Mundial & Bandas y Manchas & Simulación & (Guo et al., 2011) & Numérico \\
\hline Sudan & África & Bandas & Simulación & (Deblauwe et al., 2011) & Numérico \\
\hline Sudan & África & Bandas & Simulación & (McGrath et al., 2012) & Numérico \\
\hline Mundial & Mundial & Bandas y Manchas & Simulación & (Baek et al., 2013) & Numérico \\
\hline Mundial & Mundial & Manchas & Simulación & (Couteron et al., 2014) & Numérico \\
\hline Mundial & Mundial & Bandas & Simulación & (Chaiya et al., 2015) & Numérico \\
\hline Argentina & América & Manchas & Simulación & (Bordeu et al., 2016) & Numérico \\
\hline Mundial & Mundial & Manchas & Simulación & (Tlidi et al., 2018) & Numérico \\
\hline
\end{tabular}

Fuente: Elaboración propia

\section{Materiales y métodos}

\section{Área de estudio y condiciones generales}

Se seleccionó y caracterizó un área específica del Cabo de la Vela, zona costera de la península de La Guajira, esta área se ubica dentro del municipio de Uribía y en el resguardo indígena de la Alta Guajira, la zona es habitada en su totalidad por comunidades indígenas (Figura 2). El relieve es en un gran porcentaje plano con presencia de algunas serranías y colinas bajas, que abarcan alturas desde los 0 a los $200 \mathrm{~m}$ en su parte más alta, los habitantes se dedican al pastoreo de ungulados y a la pesca artesanal en la zona costera colindante. Tiene un área de $43 \mathrm{~km}^{2}$, en donde se tomaron muestras de la vegetación, los suelos, los parámetros ambientales y se analizaron los aspectos sociales del entorno.

Fuente: Elaboración propia con datos de Space imaging - Google Earth
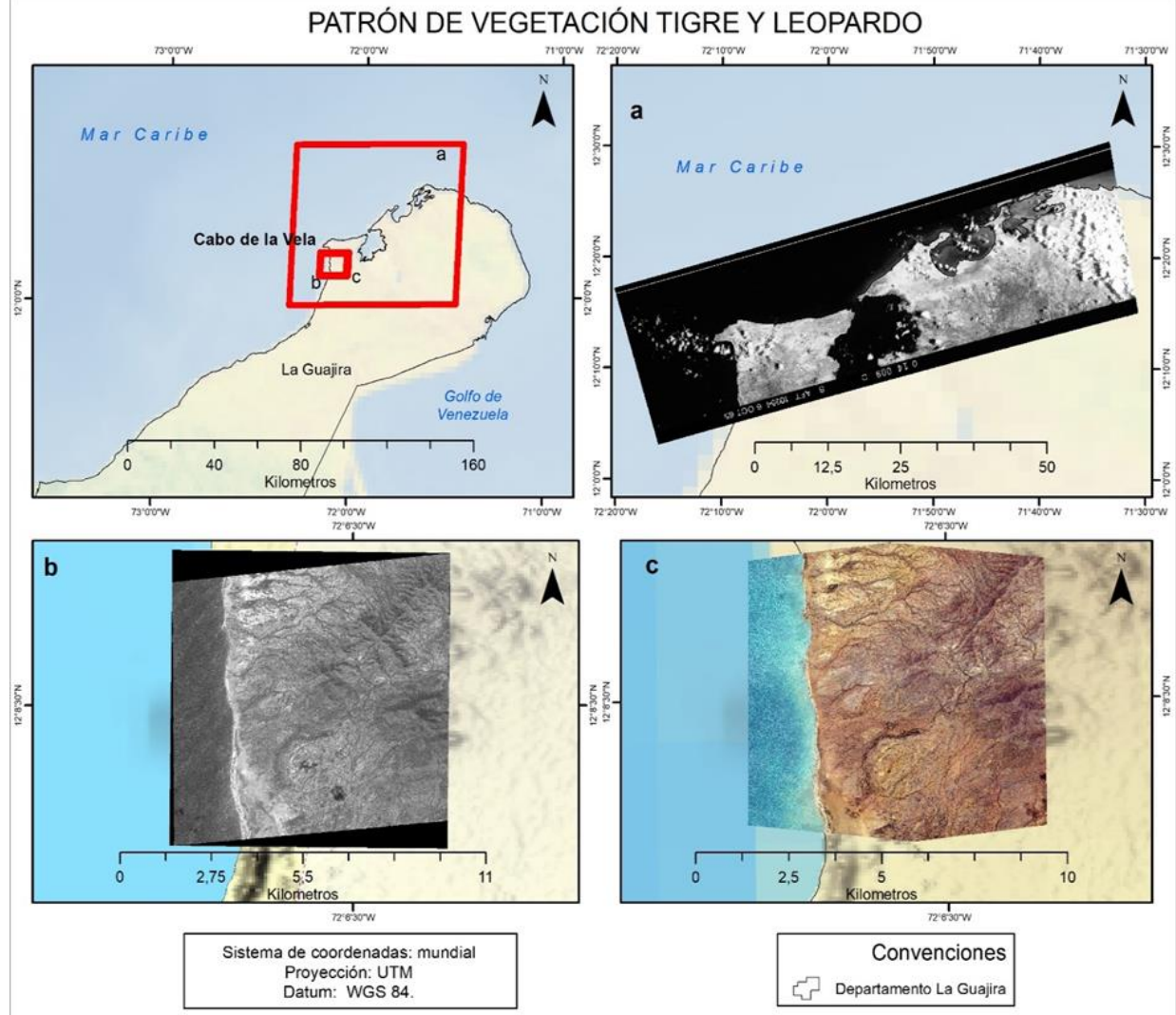

Figura 2: Imágenes de satélite utilizadas (a) Corona KH-4A 1965, (b) Eros B 2013, (c)

QuickBird 2007. En recuadro rojo se aprecia el área de estudio 


\section{Caracterización biofísica}

En el Cabo de la Vela los suelos presentes son en su mayoría Aridisoles (Typic Haplocambids, Typic Haplocalcids y Sodic Haplocambids). Son suelos jóvenes y poco evolucionados. La información edafológica general fue obtenida del Estudio General de Suelos y Zonificación de Tierras del Departamento de La Guajira del año 2009, realizado por el Instituto Geográfico Agustín Codazzi (IGAC). La información fue verificada en campo con muestras de barreno y tabla de colores Munsell para taxonomía de suelos. Para la generación del mapa geomorfológico, se utilizó una combinación de la información del DSM, el modelo de sombras, el mapa geomorfológico oficial 1:100.000 del Instituto de Investigaciones Marinas y Costeras (INVEMAR) y los datos de campo. Las características del uso del suelo como caminos, rancherías, jagüey y corrales fueron digitalizadas para todos los años con las imágenes de satélite, en la leyenda utilizada para clasificar las coberturas de la tierra se utilizaron tres entidades clasificatorias (suelo desnudo, arbustal y vegetación baja). Para desarrollar las unidades de paisaje se aplicó la metodología del análisis fisiográfico y su clasificación por el sistema CIAF - IGAC (Fuentes et al., 2020). En campo se tomaron datos de humedad de suelo con una sonda digital para tener un referente del comportamiento de esta variable en la época de lluvias (octubre), tiempo en el que fue desarrollada la salida de campo. Se realizó un análisis climático del periodo (1986 - 2013) para caracterizar el área en cuanto a su climatología. Para analizar y caracterizar los vientos del área se contó con los datos promedio de 2012 de las nueve estaciones de medición de viento de EPM.

\section{Modelo de asentamiento, evolución de la población y animales domésticos}

La distribución de las construcciones de los wayuú es dispersa, asentándose principalmente cerca de las fuentes de abastecimiento de agua (jagüey y pozos) y a lo largo de la zona plana cerca a la costa, la población también se ubica en partes más elevadas de la zona plana alejados de las áreas de influencia de los ríos que en época de lluvia generan riadas. La cantidad de personas en el área fue calculada a partir de los datos censales. También se usaron las proyecciones de población de los años 2007 y 2013 utilizó los cálculos de densidad de población a nivel municipal para luego ser extrapolados al área de estudio. En el desarrollo del modo de vida indígena la cría de cabras (Capra aegagrus hircus, Linnaeus, 1758) y ovejas (Ovis orientalis aries, Linnaeus, 1758) es la actividad económica más importante de los Wayúu. Para estimar cuantos animales existían en el área de estudio se recurrió a las estadísticas oficiales sobre los censos pecuarios de ovinos y caprinos de los años 1965, 2007, 2013 y 2016 y se extrapolaron por medio de una relación proporción área para calcular su densidad.

\section{Resultados}

\section{Determinación de distancias entre parches y composición}

Para realizar los análisis de cómo se había fragmentado la cobertura vegetal en los distintos años (1965, 2007, 2013), se utilizó el software FRAGSTATS dentro del programa ArcGis versión 10. En el área de estudio la dominancia de las coberturas según el área total (CA) fue mayoritariamente la clase suelo desnudo, esta cobertura representó en promedio el $81 \%$ del área en los últimos 48 años. Le siguió la clase arbustal, lo que representa un promedio de $18 \%$ (Tabla 2). 
Tabla 2. Métricas de cobertura vegetal área total (CA) para los diferentes años

\begin{tabular}{|c|c|c|c|c|c|c|}
\hline Cobertura & \multicolumn{2}{|c|}{$\mathbf{2 0 1 3}$} & \multicolumn{2}{c|}{$\mathbf{2 0 0 7}$} & \multicolumn{2}{c|}{1965} \\
\hline Suelo desnudo & ha & $\%$ & ha & $\%$ & ha & $\%$ \\
\hline Arbustal & 3.495 & 81.29 & 3.595 & 83.62 & 3.366 & 78.29 \\
\hline Vegetación baja & 800 & 18.6 & 692 & 16.07 & 924 & 21.49 \\
\hline Total & 4 & 0.1 & 12 & 0,28 & 9 & 0.22 \\
\hline
\end{tabular}

Fuente: Elaboración propia.

Finalmente, el valor promedio de la distancia que existe entre un fragmento y otro se pudo caracterizar utilizó el indicie Mean Euclidean Nearest Neighbor Distance (ENN_MN). Para la clase arbustal el año 1965 inició con una distancia de $8 \mathrm{~m}$ entre los fragmentos para luego pasar de $4 \mathrm{~m}$ en el año 2007 y finalmente $3 \mathrm{~m}$ en 2013, métrica que coincide con los datos de algunas mediciones en terreno de 2014, la clase suelos desnudos evolucionó de 7 m en promedio a 4 m en 2007 y se mantuvo en esa cifra hasta el 2013 al igual que la vegetación baja que en el año 1965 tenía $15 \mathrm{~m}$ de distancia y se redujo a $6 \mathrm{~m}$ en los siguientes años. Con la vegetación que se encontró en el momento de la salida de campo se realizaron perfiles para mostrar las comunidades y el modelo de sucesión con relación a un corte topográfico. La vegetación es el principal elemento dentro del desarrollo del patrón Leopardo, en campo se logró la identificación de once especies de flora. Se encontraron las siguientes especies (Tabla 3). Un aspecto relacionado fuertemente con el paisaje fue la agrupación de la vegetación, al encontrarse dos comunidades de especies vegetales que principalmente conforman los parches (Comunidad Heterostachys ritteriana - Sesuvium portulacastrum y Comunidad Castela erecta - Prosopis juliflora - Opuntia caracasanan).

Tabla 3. Listado de especies identificadas.

\begin{tabular}{|c|c|c|c|}
\hline Familia & Genero & Especie & Autor \\
\hline Simaroubaceae & Castela & Castela erecta & (Turpin) \\
\hline Fabaceae & Prosopis & Prosopis juliflora & (Sw.) DC \\
\hline Fabaceae & Parkinsonia & Parkinsonia praecox & (Ruiz \& Pav.) Hawkins \\
\hline Cactaceae & Melocactus & Melocactus curvispinus & (Pfeiff) \\
\hline Cactaceae & Opuntia & Opuntia caracasana & (Salm) Dyck, \\
\hline Cactaceae & Pilosocereu & $\begin{array}{c}\text { Pilosocereus } \\
\text { lanuginosus }\end{array}$ & (L.) Byles \& \\
& & G.D.Rowley, \\
\hline Cactaceae & Stenocereus & Sesuvium & (Haw.) Buxb, \\
\hline Aizoaceae & Sesuvium & portulacastrum & (L.), \\
\hline Euphorbiaceae & Jatropha & Jatropha gossypiifolia & (L.) \\
\hline Amaranthaceae & Heterostachys & Heterostachys & (Moq.) Ung.-Sternb \\
& & ritteriana & (L.). \\
\hline Portulacaceae & Portulaca & Portulaca halimoides & (Turpin) \\
\hline Simaroubaceae & Castela & Castela erecta & (Sw.) DC \\
\hline Fabaceae & Prosopis & Prosopis juliflora & \\
\hline
\end{tabular}

Fuente: Elaboración propia.

\section{Dinámica de nutrientes en el suelo}

Las medidas de $\mathrm{N}, \mathrm{P}, \mathrm{K}$ tuvieron como unidad $1 \mathrm{ppm}=1 \mathrm{mg} / \mathrm{kg}$, la humedad se midió en variación de 0 a 9 según el instrumento. Luego de separar y organizar las muestras, la primera característica 
que se analizó fue el pH, este arrojó un promedio de 7,38 lo que ubicó las muestras en el rango de básico. Seguido al análisis del pH, se comenzó con las pruebas sobre los macronutrientes N, P, K. Para el elemento N, la mayoría de las estaciones mostraron una tendencia a que los valores dentro de la vegetación fueran ligeramente superiores mostrando que este elemento varía en función de la cobertura. Los valores en general fueron clasificados como bajos con 16 ppm. El macronutriente P, arrojó resultados muy parecidos en todas las estaciones comportándose en la misma forma que el $\mathrm{N}$ al demostrar variaciones en los lugares vegetados con incrementos en los valores a diferencia de los lugares no vegetados con valores más bajos. El P en promedio se clasificó como medio - alto con 71 ppm un valor que indica la disponibilidad de este nutriente para las plantas en el área. El Potasio (K) tuvo un comportamiento similar a los otros elementos incremento los niveles en las áreas vegetadas y descendiendo en las áreas sin vegetación. En general el valor fue bajo con 153 ppm en promedio lo que indica baja disponibilidad. Por medio de la sonda de humedad se pudo verificar la existencia de un aumento en la humedad del suelo dentro de la vegetación y una disminución en los lugares donde el suelo se encontraba desnudo. Los valores promedio dentro de la vegetación se ubicaron en el valor neutro de humedad. Los valores promedio para el suelo desnudo fueron de 0 teniendo un valor bajo de humedad, teniendo en cuenta que el momento de la toma de datos fue el final del periodo húmedo en octubre.

\section{Fragmentación, presión antrópica y animal}

$\mathrm{Al}$ analizar cómo había sido la evolución de la cobertura vegetal en el área de estudio, se evidencio que a través del tiempo la cobertura ha venido disminuyendo frente al aumento de los suelos desnudos. Al evaluar los índices de fragmentación, éstos mostraron la continua disminución en la cobertura, y el aumento en la cantidad de los fragmentos de arbustal, la disminución de las distancias entre parches y la reducción del tamaño de los parches de vegetación evidenciándose el efecto antrópico en los caminos resultantes por el tránsito de vehículo y del trasegar de los animales. La población en el área aumentó de manera acorde al crecimiento poblacional de la región y del país teniendo un incremento alrededor de nueve veces en 2013 (855 personas) comparado con la población inicial en el año 1964 (90 personas). La evolución de las construcciones, áreas de corrales y cuerpos de agua mostro la tendencia al aumento progresivo de la colonización en el área, contrastado con el aumento de la población humana y animal. Con las imágenes de satélite de los distintos años se logró digitalizar la información correspondiente a caminos, infraestructura y las acumulaciones de agua (jaguey) presentes en cada una de las imágenes de los distintos años. El ganado en el área se incrementó siete veces desde 1965 al 2007. De 2007 a 2013 se duplicó la cantidad de animales, pasó de 1334 a 3063 para posteriormente seguir con un incremento constante hacia el año 2016 con un valor de 3141, lo que refleja el grado de incremento anual en la presión del ganado sobre la vegetación del área, se observa también una clara tendencia a que los caprinos superen al ganado ovino, probablemente debido a que las cabras son más resistentes a las sequías y son capaces de soportar mejor estos cambios.

Las coberturas fueron afectadas principalmente para crear caminos y senderos por donde transitan las personas, bicicletas, motos y vehículos. Al analizar la evolución de los caminos y las diferencias a través de los años se evidenció la cantidad que existen en el área de estudio (324 km en 2013) distribuyéndose ampliamente sobre toda la zona con excepción de las partes altas. El elemento que 
generó la mayor perturbación en la vegetación es el pastoreo, animales domesticados tienen comportamientos que conducen a la heterogeneidad de la vegetación, al seguir un patrón de movimiento que genera rutas regulares alrededor de los puntos de agua, descanso y alimento. El principal factor que determina la dispersión de animales por la zona es el relieve, lo que ayuda a que se dispersen por toda el área en pequeños grupos. Con las imágenes de satélite se pudo constatar que los asentamientos wayuú están dispersos en el espacio, a medida de los años han aumentado y en algunos casos han desaparecido o se han modificado sustancialmente. De acuerdo con Arnold et al., (1978) "en cuanto más deteriorada es la pastura más subgrupos son formados para realizar el pastoreo lo que con lleva un nivel de separación mayor" (pp. 8). El otro elemento que interviene en el proceso de la fragmentación vinculado a los animales es el efecto del pisoteo sobre el suelo. Esta compactación hace que se pierdan los nutrientes reduciendo en gran medida los aportes de nitrógeno y carbono, también aumenta la impermeabilidad reduciendo la disponibilidad de agua al disminuir la infiltración. Este efecto también se desarrolla con los caminos creados por los humanos.

\section{Islas de fertilidad y formación del Patrón Leopardo}

El efecto de la humedad general en el suelo fue relacionado espacialmente interceptando el índice de humedad topográfico (calculado desde el modelo de superficie) y el mapa de coberturas del año 2013 para luego ser reclasificado, este mostró una relación espacial en donde los parches de vegetación que conforman el patrón Leopardo tienden a asentarse principalmente en los lugares por donde puede escurrir o acumular el agua a nivel topográfico. Dentro de los parches y las distintas especies individuales afines al patrón Leopardo, se pudo encontrar evidencia de un fenómeno muy común en zonas áridas, este fenómeno es llamado isla de fertilidad o isla de recursos, el cual consiste en la creación de un microhábitat que permite un desarrollo más favorable del ecosistema presente dentro de la isla, en contraposición a las zonas que no presentan el fenómeno (suelo desnudo).

Estas islas generan cambios en el microclima y en las propiedades del suelo agrupando una mayor concentración de nutrientes y diversidad microbiana, durante el trabajo de campo se pudo constatar la existencia de dos tipos de islas de recursos que integran procesos tanto físicos como biológicos y en las cuales se conforman los patrones de la vegetación Leopardo. La primera forma de isla "micro isla" es conformada por plantas individuales (Figura 3a) principalmente arbustos (Heterostachys ritteriana, Castela erecta y Prosopis juliflora) que acumulan una capa o montículo (micro duna). Esto se forma a partir de la deposición y posterior estabilización de las partículas del polvo transportadas por el viento y retenidas por la rugosidad que estas plantas generan bajo su dosel, la micro duna mejoró las características locales del suelo y dio lugar a mayores niveles de nutrientes (NPK), y aumentó la infiltración en el suelo en los periodos lluviosos, esto se pudo corroborar en los análisis de macronutrientes y humedad dentro de la vegetación. Mientras que los espacios adyacentes sin vegetación son relativamente más estériles y carentes de actividad biótica. 

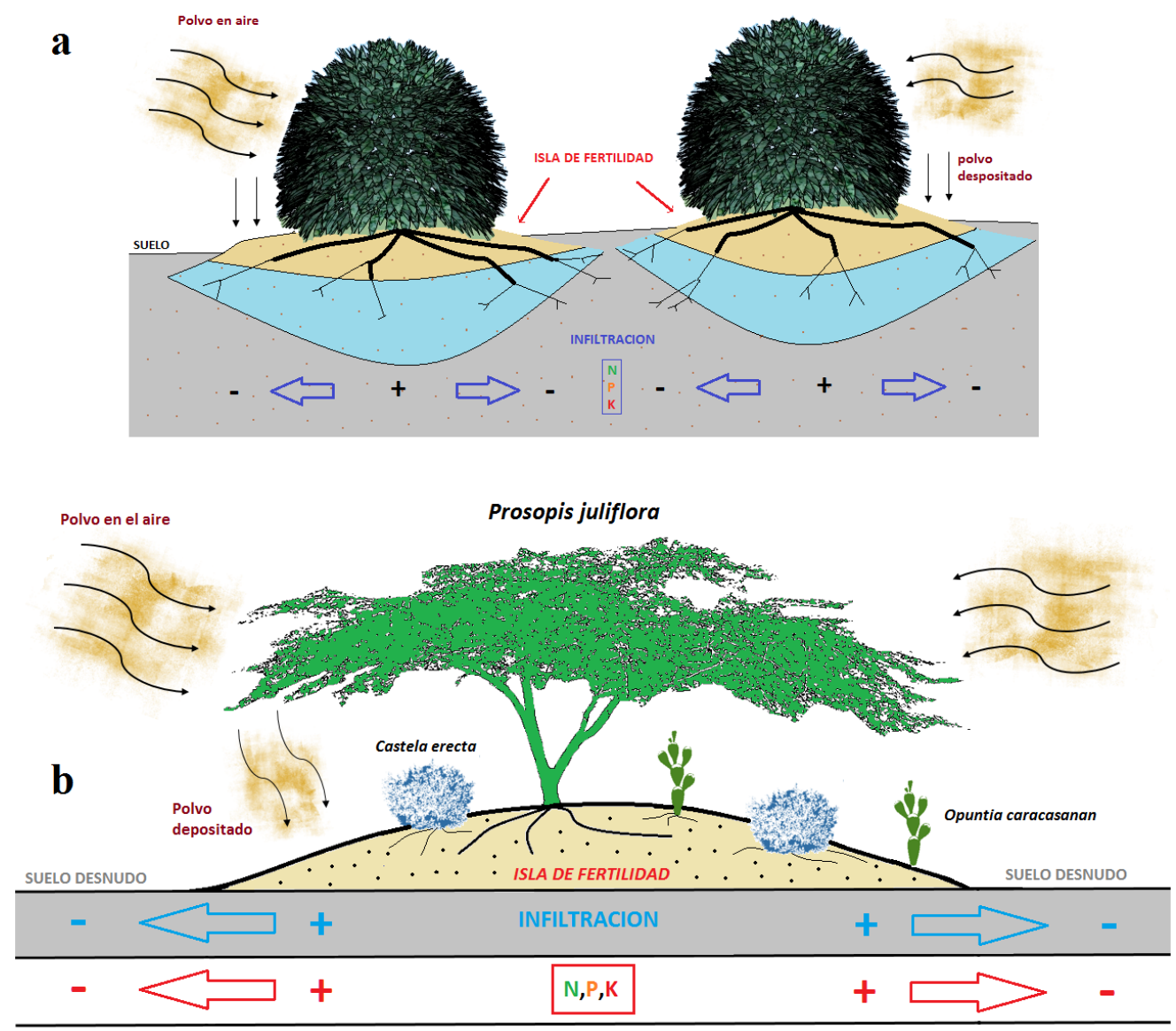

Figura 3: Esquema de la formación de micro (a) y macro (b) islas de fertilidad dentro del patrón Leopardo

La segunda formación de la isla de fertilidad, fue la "macro isla", éste es un hábitat que facilita el establecimiento de varias especies, el desarrollo de la macro isla comienza con una planta ancla (facilitación), generalmente un arbusto o árbol que crea un efecto sombra (Prosopis juliflora) que incrementa la riqueza de especies que se refugian bajo su dosel al permitir que las semillas y plántulas de otras especies se desarrollen bajo su efecto de sombrilla. Debajo de la planta ancla se encuentran generalmente cactáceas (Opuntia caracasanan) y arbustos (Castela erecta) alrededor o dentro de la zona de las raíces y ramas colgantes de la especie ancla, cada planta ayuda a la formación de montículos (micro duna) de suelo por deposición (polvo) y en conjunto con la planta ancla estabilizan las partículas llevadas por el viento bajo el dosel y alrededor de esta. A su vez este proceso facilita el establecimiento de otras especies incrementando la riqueza dentro de la isla (Figura 3b). En los datos analizados en las islas de fertilidad, se encontró que los valores de nutrientes (N P K) y la humedad fueron superiores en comparación con los de los suelos desnudos. Durante la época de lluvias, las semillas (gramíneas y leñosas) atrapadas en la micro duna germinan debido al aumento de la humedad. Dentro de las islas de fertilidad los valores de los 
macronutrientes son mayores que los de los suelos desnudos circundantes, también se incrementa la infiltración debido a la presencia de las plantas. Durante la temporada de lluvias, la erosión hídrica redistribuye el material superficial y durante la estación seca, los suelos compactados y los espacios fragmentados por la influencia antrópica, que contienen sedimentos arenosos, son eliminados por el viento dejando los suelos desnudos entre los parches de vegetación conformando así el patrón Leopardo

\section{Discusión}

La presión del pastoreo de ungulados fue un resultado que se mencionó en la literatura (Elwell \& Stocking, 1976; Kelly \& Walker, 1976; Breman \& Wit, 1983; Stroosnijder, 1996; Aguiar \& Sala, 1999; Fuentes et al., 2018). El pastoreo y pisoteo intenso del ganado en las regiones semiáridas, concentrado alrededor de lugares de riego, a menudo conduce a una reducción de la cubierta vegetal y a la compactación del suelo. El pastoreo excesivo puede alterar las relaciones in situ planta-suelo y crear heterogeneidad espacial (Schlesinger et al., 1990; Schlesinger \& Pilmanis, 1998). Kellner y Bosch, (1992) sugirieron que los patrones de vegetación en praderas semiáridas se crearon mediante el pastoreo selectivo por los herbívoros. Dunkerley \& Brown, (1995) en los estudios del patrón Tigre en Australia, mencionan la relación con la formación de patrones con las bandas de vegetación. "Las bandas evolucionan a medida que se adelgaza una cobertura vegetal densa por deterioro climático o por la presión del pastoreo" (pp. 3). Estos estudios reconocen que el pastoreo y las actividades antrópicas pueden modificar los patrones de vegetación. Uno de los resultados que es consecuente con otros autores es la mayor humedad en las zonas donde está presente la vegetación. La variación espacial resultante puede estructurarse en ciertas escalas, donde la vegetación en parches concentra nutrientes y agua en el suelo, pero son eliminados en los parches de suelo desnudo o compactado (Tongway \& Ludwig, 1990; Schlesinger \& Pilmanis, 1998; Valentin \& d'Herbès, 1999; Aguiar \& Sala, 1999; Wilson, 2000; Menaut et al., 2001; Ursino, 2005; Saco et al., 2007). Respecto a la influencia del viento y la diseminación de las semillas en la formación del patrón Leopardo, Aguiar \& Sala, (1999) especificaron algo que se corroboró en La Guajira: el agua es el principal agente de la redistribución de la vegetación Tigre, mientras que el viento y los animales son la principal causa de la redistribución de la vegetación de Leopardo.

Los resultados mostraron características diferenciales en el patrón Leopardo presente en La Guajira. La zona se ubica en un promedio anual con un máximo de precipitación de $100 \mathrm{~mm}$, este es el primer aspecto diferencial entre el patrón Leopardo y el patrón Tigre, debido a que este último según algunos autores (Valentin \& d'Herbès, 1999; Menaut et al., 2001) puede encontrarse en rangos de precipitación desde los $300 \mathrm{~mm}$ a los $800 \mathrm{~mm}$.

Queda claro que la relación entre los patrones en bandas y rayas son diferenciables de primera mano por medio de la precipitación promedio tendiendo a ser inferior a los $300 \mathrm{~mm}$ en el patrón Leopardo. En el estudio del patrón Leopardo los análisis sobre la vegetación mostraron que la vegetación se compone de herbáceas, arbustales y cactáceas, estas últimas son uno de los resultados más significativos debido a que este sería el primer reporte donde los cactus forman parte del patrón Leopardo.

\section{Conclusiones}

La composición de la vegetación dentro de estos parches mostró la tendencia a la dominancia de 
los arbustos como la Castela erecta y Prosopis juliflora, presentes en la mayoría de los parches y con gran dominancia sobre las asociaciones de la vegetación en la zona de estudio. Al realizar las pruebas de campo sobre los suelos, fue posible conocer las interacciones de las plantas con el viento, el resultado de esta interacción mostró la influencia que las corrientes de aire locales tienen sobre los parches, en donde se comprobó las estrategias que la vegetación adoptaba formando islas de fertilidad para lograr una mejora del ecosistema circundante. En campo se pudo probar esta diferencia al comparar la medición de los nutrientes presentes en los suelos circundantes a la vegetación y los espacios no vegetados, arrojo mayores valores más significativos en la mayoría de los lugares vegetados que en los no vegetados. Esto demostró que la heterogeneidad del paisaje y por tanto del patrón es debida a esta variación de nutrientes forman espacios alternados de disponibilidad de recursos fragmentados principalmente por la acción humana y animal.

\section{Referencias Bibliográficas}

Aguiar, M., \& Sala, O. E. (1999). Patch structure, dynamics and implications for the functioning of arid ecosystems. Trends in Ecology \& Evolution, 14(7), 273-277. https://doi.org/10.1016/S0169-5347(99)01612-2

Arnold, G., Arnold, M., \& Dudzinsky, M. (1978). Ethology of free-ranging domestic animals. Journal of Mammalogy, 63(4), 720. https://doi.org/10.2307/1380297

Baek, H., Jung, D. I., \& Wang, Z. (2013). Pattern formation in a semi-ratio-dependent predatorprey system with diffusion. Discrete Dynamics in Nature and Society, 2013, 14. https://doi.org/10.1155/2013/657286

Barbier, N., Couteron, P., Lefever, R., Deblauwe, V., \& Lejeune, O. (2008). Spatial decoupling of facilitation and competition at the origin of gapped vegetation patterns. Ecology, 89(6), 1521-1531. https://doi.org/10.1890/07-0365

Bestelmeyer, B., Trujillo, D., Tugel, A., \& Havstad, K. (2006). A multi-scale classification of vegetation dynamics in arid lands: What is the right scale for models, monitoring, and restoration? Journal of Arid Environments, 65(2), 296-318. https://doi.org/10.1016/j.jaridenv.2005.06.028

Blyth, E., \& Harding, R. (1995). Application of aggregation models to surface heat flux from the Sahelian tiger bush. Agricultural and Forest Meteorology, 72(3-4), 213-235. https://doi.org/10.1016/0168-1923(94)02164-F

Boaler, S., \& Hodge, C. (1962). Vegetation stripes in Somaliland. The Journal of Ecology, 50 (2), 465-474. https://doi.org/10.2307/2257457

Bordeu, I., Clerc, M. G., Couteron, P., Lefever, R., \& Tlidi, M. (2016). Self-Replication of Localized Vegetation Patches in Scarce Environments. Scientific Reports, (6), 1-11. https://doi.org/10.1038/srep33703

Borgogno, F., D'Odorico, P., Laio, F., \& Ridolfi, L. (2009). Mathematical models of vegetation pattern formation in ecohydrology. Reviews of geophysics, 47(1), 1-36. https://doi.org/10.1029/2007RG000256

Borthagaray, A. I., Fuentes, M. A., \& Marquet, P. A. (2010). Vegetation pattern formation in a fogdependent ecosystem. Journal of theoretical biology, 265(1), 18-26. 
https://doi.org/10.1016/j.jtbi.2010.04.020

Breman, H., \& Wit, C. T. d. (1983). Rangeland productivity and exploitation in the Sahel. Science, 221(4618), 1341-1347. https://doi.org/10.1126/science.221.4618.1341

Chaiya, I., Wollkind, D. J., Cangelosi, R. A., Kealy-Dichone, B. J., \& Rattanakul, C. (2015). Vegetative Rhombic Pattern Formation Driven by Root Suction for an InteractionDiffusion Plant-Ground Water Model System in an Arid Flat Environment. American Journal of Plant Sciences, 6, 1278-1300. http://dx.doi.org/10.4236/ajps.2015.68129

Cheng, Y., Stieglitz, M., Engel, V., \& Turk, G. (2010). Parallel Vegetation Stripe Formation Through Hydrologic Interactions.

Couteron, P., Anthelme, F., Clerc, M., Escaff, D., Fernandez-Oto, C., \& Tlidi, M. (2014). Plant clonal morphologies and spatial patterns as self-organized responses to resource-limited environments. Philosophical Transactions of the Royal Society A: Mathematical, Physical and Engineering Sciences, 372(2027), 17. https://doi.org/10.1098/rsta.2014.0102

Couteron, P., \& Lejeune, O. (2001). Periodic spotted patterns in semi-arid vegetation explained by a propagation-inhibition model. Journal of Ecology, 89(4), 616-628. https://doi.org/10.1046/j.0022-0477.2001.00588.x

Deblauwe, V., Couteron, P., Lejeune, O., Bogaert, J., \& Barbier, N. (2011). Environmental modulation of self-organized periodic vegetation patterns in Sudan. Ecography, 34(6), 9901001. doi/epdf/10.1111/j.1600-0587.2010.06694.x

Dunkerley, D. (1997). Banded vegetation: survival under drought and grazing pressure based on a simple cellular automaton model. Journal of Arid Environments, 35(3), 419-428. https://doi.org/10.1006/jare.1995.0158

Dunkerley, D. L., \& Brown, K. J. (1995). Runoff and runon areas in a patterned chenopod shrubland, arid western New South Wales, Australia: characteristics and origin. Journal of Arid Environments, 30(1), 41-55. https://doi.org/https://doi.org/10.1016/S01401963(95)80037-9

Elwell, H. A., \& Stocking, M. A. (1976). Vegetal cover to estimate soil erosion hazard in Rhodesia. Geoderma, 15(1), 61-70. https://doi.org/10.1016/0016-7061(76)90071-9

Fuentes, J., Varga, D., y Boada, M. (2016). Distribución del patrón espacial tipo leopardo en regiones áridas y semiáridas del mundo. Boletín de la Asociación de Geógrafos Españoles, (71), 59-72. https://doi.org/10.21138/bage.2274

Fuentes, J., Varga, D., \& Pintó, J. (2018). The Use of High-Resolution Historical Images to Analyse the Leopard Pattern in the Arid Area of La Alta Guajira, Colombia. Geosciences, 8(10), 366. https://doi.org/10.3390/geosciences8100366

Fuentes, J. E., Moya, F. D., \& Montoya, O. D. (2020). Method for Estimating Solar Energy Potential Based on Photogrammetry from Unmanned Aerial Vehicles. Electronics, 9(12), 2144. https://doi.org/10.3390/electronics9122144

Garcia-Moya, E., \& McKell, C. M. (1970). Contribution of shrubs to the nitrogen economy of a desert-wash plant community. Ecology, 51(19), 81-88. https://doi.org/10.2307/1933601

Gilad, E., von Hardenberg, J., Provenzale, A., Shachak, M., \& Meron, E. (2004). Ecosystem engineers: from pattern formation to habitat creation. Physical Review Letters, 93(9). https://doi.org/10.1103/PhysRevLett.93.098105 
Guo, T., Tan, Q., \& Xiong, J. (2011). Analysis of spatial patterns in a vegetation model. Applied Mathematics and Computation, 217(21), 8303-8310. https://doi.org/10.1016/j.amc.2011.03.012

HilleRisLambers, R., Rietkerk, M., van den Bosch, F., Prins, H. H., \& de Kroon, H. (2001). Vegetation pattern formation in semi-arid grazing systems. Ecology, 82(1), 50-61. https://doi.org/10.2307/2680085

Instituto de Hidrología, Meteorología y Estudios Ambientales. IDEAM. (2005). Atlas Climático de Colombia. Bogotá. https://bit.ly/3uBwhzX

Kefi, S., Rietkerk, M., \& Katul, G. G. (2008). Vegetation pattern shift as a result of rising atmospheric CO 2 in arid ecosystems. Theoretical population biology, 74(4), 332-344. https://doi.org/10.1016/j.tpb.2008.09.004

Kellner, K., \& Bosch, O. (1992). Influence of patch formation in determining the stocking rate for southern African grasslands. Journal of Arid Environments, 22(1), 99-105. https://doi.org/10.1016/S0140-1963(18)30661-X

Kelly, R. D., \& Walker, B. H. (1976). The Effects of Different Forms of Land Use on the Ecology of a Semi-Arid Region in South-Eastern Rhodesia. Journal of Ecology, 64(2), 553-576. https://doi.org/10.2307/2258773

Lefever, R., \& Lejeune, O. (1997). On the origin of tiger bush. Bulletin of Mathematical biology, (59), 263-294. https://doi.org/10.1007/BF02462004

Lejeune, O., Couteron, P., \& Lefever, R. (1999). Short range co-operativity competing with long range inhibition explains vegetation patterns. Acta Oecologica, 20(3), 171-183. https://doi.org/10.1016/S1146-609X(99)80030-7

Liu, Q.-X., Jin, Z., \& Li, B.-L. (2008). Numerical investigation of spatial pattern in a vegetation model with feedback function. Journal of theoretical biology, 254(2), 350-360. https://doi.org/10.1016/j.jtbi.2008.05.017

MacArthur, R. H., y Wilson, E. (1967). The theory of island biogeography. Princeton; Oxford: Princeton University Press.

Macfadyen, W. (1950). Vegetation patterns in the semi-desert plains of British Somaliland. The Geographical Journal, 116(4/6), 199-211. https://doi.org/10.2307/1789384

Manor, A., \& Shnerb, N. M. (2008). Facilitation, competition, and vegetation patchiness: from scale free distribution to patterns. Journal of theoretical biology, 253(4), 838-842. https://doi.org/10.1016/j.jtbi.2008.04.012

McGrath, G. S., Paik, K., \& Hinz, C. (2012). Microtopography alters self-organized vegetation patterns in water-limited ecosystems. Journal of Geophysical Research: Biogeosciences, 117(G3). https://doi.org/10.1029/2011JG001870

Meigs, P. (1953). World Distribution of Arid and Semi-arid Hot Climates. Paris: UNESCO. https://bit.ly/2RHjgaB

Menaut, J.-C., Walker, B., Tongway, D. J., Valentin, C., \& Seghieri, J. (2001). Banded vegetation patterning in arid and semiarid environments. Ecological processes and consequences for management 149.

Rietkerk, M., Ketner, P., Burger, J., Hoorens, B., \& Olff, H. (2000). Multiscale soil and vegetation patchiness along a gradient of herbivore impact in a semi-arid grazing system in West 
Africa. Plant Ecology, 148(2), 207-224. https://doi.org/10.1023/A:1009828432690

Rietkerk, M., \& Van de Koppel, J. (2008). Regular pattern formation in real ecosystems. Trends in Ecology \& Evolution, 23(3), 169-175. https://doi.org/10.1016/j.tree.2007.10.013

Saco, P., Willgoose, G., \& Hancock, G. (2007). Eco-geomorphology of banded vegetation patterns in arid and semi-arid regions. Hydrology and Earth System Sciences, 11(6), 1717-1730. https://doi.org/10.5194/hess-11-1717-2007

Sala, O. E., \& Aguiar, M. R. (1996). Origin, maintenance, and ecosystem effect of vegetation patches in arid lands. Proceedings Vth International Rangeland Congress, Salt Lake City, Utah.

Schlesinger, W. H., \& Pilmanis, A. M. (1998). Plant-soil Interactions in Deserts. Biogeochemistry, 42(1/2), 169-187. https://doi.org/10.1023/a:1005939924434

Schlesinger, W. H., Reynolds, J. F., Cunningham, G. L., Huenneke, L., Jarrell, W., Virginia, R., \& Whitford, W. (1990). Biological feedbacks in global desertification. Science, 247(4946), 1043-1048. 10.1126/science.247.4946.1043

Slatyer, R. O. (1959). Methodology of a water balance study conducted on a desert woodland (acacia aneura f. muell.) community in central Australia Symposium on Plant-Water Relationships in Arid and Semi-arid Conditions, Madrid.

Stroosnijder, L. (1996). Modelling the effect of grazing on infiltration, runoff and primary production in the Sahel. Ecological modelling, 92(1), 79-88. https://doi.org/10.1016/03043800(95)00197-2

Tlidi, M., Bordeu, I., Clerc, M. G., \& Escaff, D. (2018). Extended patchy ecosystems may increase their total biomass through self-replication. Ecological Indicators, 94, 534-543. https://doi.org/10.1016/j.ecolind.2018.02.009

Tongway, D. J., \& Ludwig, J. A. (1990). Vegetation and soil patterning in semi-arid mulga lands of Eastern Australia. Austral Ecology, 15(1), 23-34. https://doi.org/10.1111/j.14429993.1990.tb01017.x

Ursino, N. (2005). The influence of soil properties on the formation of unstable vegetation patterns on hillsides of semiarid catchments. Advances in water resources, 28(9), 956-963. https://doi.org/10.1016/j.advwatres.2005.02.009

Valentin, C., \& d'Herbès, J.-M. (1999). Niger tiger bush as a natural water harvesting system. Catena, 37(1-2), 231-256. https://doi.org/10.1016/S0341-8162(98)00061-7

Wickens, G. E., \& Collier, F. W. (1971). Some vegetation patterns in the Republic of the Sudan. Geoderma, 6(1), 43-59. http://dx.doi.org/10.1016/0016-7061(71)90050-4

Wilson, J. P., \& Gallant, J.C. (2000). Terrain analysis: principles and applications. John Wiley \& Sons.

Worrall, G. (1959). The Butana grass patterns. European Journal of Soil Science, 10(1), 34-53. https://doi.org/10.1111/j.1365-2389.1959.tb00664.x

Yetemen, O., Istanbulluoglu, E., \& Vivoni, E. R. (2010). The implications of geology, soils, and vegetation on landscape morphology: Inferences from semi-arid basins with complex vegetation patterns in Central New Mexico, USA. Geomorphology, 116(3-4), 246-263. https://doi.org/10.1016/j.geomorph.2009.11.026 